ACCEPTED to ApJ

Preprint typeset using $\mathrm{LT}_{\mathrm{E}} \mathrm{X}$ style emulateapj v. 11/10/09

\title{
A RELATIONSHIP BETWEEN AGN JET POWER AND RADIO POWER
}

\author{
K. W. Cavagnolo ${ }^{1,6}$, B. R. McNamara ${ }^{1,2,3}$, P. E. J. Nulsen ${ }^{3}$, \\ C. L. CARILLI ${ }^{4}$, C. JONES ${ }^{3}$, AND L. BÎRZAN ${ }^{5}$, \\ Accepted to ApJ
}

\begin{abstract}
Using Chandra X-ray and VLA radio data, we investigate the scaling relationship between jet power, $P_{\text {jet }}$, and synchrotron luminosity, $P_{\text {radio }}$. We expand the sample presented in Bîrzan et al. (2008) to lower radio power by incorporating measurements for $21 \mathrm{gEs}$ to determine if the Bîrzan et al. (2008) $P_{\text {jet }}-P_{\text {radio }}$ scaling relations are continuous in form and scatter from giant elliptical galaxies (gEs) up to brightest cluster galaxies (BCGs). We find a mean scaling relation of $P_{\text {jet }} \approx 5.8 \times 10^{43}\left(P_{\text {radio }} / 10^{40}\right)^{0.70} \mathrm{erg} \mathrm{s}^{-1}$ which is continuous over $\sim 6-8$ decades in $P_{\text {jet }}$ and $P_{\text {radio }}$ with a scatter of $\approx 0.7$ dex. Our mean scaling relationship is consistent with the model presented in Willott et al. (1999) if the typical fraction of lobe energy in non-radiating particles to that in relativistic electrons is $\gtrsim 100$. We identify several gEs whose radio luminosities are unusually large for their jet powers and have radio sources which extend well beyond the densest parts of their X-ray halos. We suggest that these radio sources are unusually luminous because they were unable to entrain appreciable amounts of gas.
\end{abstract}

Subject headings: galaxies: active - galaxies: clusters: general - X-rays: galaxies - radio continuum: galaxies

\section{INTRODUCTION}

Most galaxies harbor a central supermassive black hole $(\mathrm{SMBH})$ which likely co-evolved with the host galaxy, giving rise to correlations between bulge luminosity, stellar velocity dispersion, and central black hole mass (Kormendy \& Richstone 1995; Magorrian et al. 1998). Models suggest these correlations were imprinted via galaxy mergers and the influence of feedback from active galactic nuclei (AGN) (e.g. Silk \& Rees 1998; Kauffmann \& Haehnelt 2000). Around the time of these discoveries, the $C X O$ found direct evidence for AGN feedback when observations revealed cavities and shock fronts in the X-ray emitting gas surrounding many massive galaxies (e.g. Fabian et al. 2000; McNamara et al. 2000). X-ray cavities provide a direct measurement of the mechanical energy released by AGN through work done on the hot, gaseous halos surrounding them (McNamara et al. 2000). This energy is expected to heat the gas (Churazov et al. 2001) and prevent it from cooling and forming stars

Studies of X-ray cavities have shown that AGN feedback supplies enough energy to regulate star formation and suppress cooling of the hot halos of galaxies and clusters (Bîrzan et al. 2004; Dunn et al. 2005; Rafferty et al. 2006). Further supported by numerical simulations (e.g. Croton et al. 2006; Bower et al. 2006), a consensus has emerged that AGN feedback plays an important role in regulating galaxy evolution at late times. However, the details of how AGN feedback is coupled to the thermodynamics of the host cluster is still uncertain (De Young et al. 2008; Mathur et al. 2009). Studying

\footnotetext{
${ }^{1}$ Department of Physics \& Astronomy, University of Waterloo, 200 University Ave. W., Waterloo, Ontario, N2L 3G1, Canada.

${ }^{2}$ Perimeter Institute for Theoretical Physics, 31 Caroline St. N., Waterloo, Ontario, N2L 2Y5, Canada.

${ }^{3}$ Harvard-Smithsonian Center for Astrophysics, 60 Garden St., Cambridge, Massachusetts, 02138-1516, United States.

${ }^{4}$ National Radio Astronomy Observatory, P.O. Box 0, Socorro, NM 87801-0387, United States.

${ }^{5}$ Leiden Observatory, University of Leiden, P.O. 9513, 2300 RA Leiden, The Netherlands.

${ }^{6}$ kcavagno@uwaterloo.ca
}

feedback in the broader cosmological context can be accomplished in principle using radio observations to trace AGN activity (e.g. Best et al. 2007). In order to do so, scaling relationships between radio luminosity and mechanical jet power are required to estimate $P_{\text {jet }}$ in systems where X-ray observations of their halos are either lacking or infeasible.

Relationships between $P_{\text {jet }}$ and $P_{\text {radio }}$ were presented in Bîrzan et al. (2004, hereafter B04) and Bîrzan et al. (2008, hereafter B08). In B08, scaling relations between $P_{\text {jet }}$ and 327 $\mathrm{MHz}, 1.4 \mathrm{GHz}$, and bolometric radio luminosities were discussed. B08 found $P_{\text {jet }} \propto P_{\text {radio }}^{0.5-0.7}$ depending on the observed radio frequency. However, there are few objects in the B08 study with $P_{\text {radio }} \lesssim 10^{38} \mathrm{erg} \mathrm{s}^{-1}$ and $P_{\text {jet }} \lesssim 10^{43} \mathrm{erg} \mathrm{s}^{-1}$, and the relations have rather large scatter (discussed in Section 4.1). In this paper we extend the study of B08 with the inclusion of $21 \mathrm{gEs}$ from systems with lower X-ray luminosities and jet powers compared to those found in rich clusters.

We outline the sample of gEs in $\S 2$. X-ray and radio measurements are discussed in $\S 3$. Results and discussion are presented in $\$ 4$. The summary and concluding remarks are given in $\S 5$. A $\Lambda$ CDM cosmology with $H_{0}=70 \mathrm{~km} \mathrm{~s}^{-1} \mathrm{Mpc}^{-1}$, $\Omega_{\mathrm{M}}=0.27$, and $\Omega_{\Lambda}=0.73$ is adopted. All quoted uncertainties are $68 \%$ confidence.

\section{SAMPLE}

The $21 \mathrm{gEs}$ in this study (see Table 1) were taken from the sample of $160 \mathrm{gEs}$ compiled by Jones et al. (in preparation). The Jones et al. compilation is drawn from the samples of Beuing et al. (1999) and O'Sullivan et al. (2003) using the criteria that the $K$-band luminosity exceeds $10^{10} L_{\odot}$ and the object has been observed with Chandra. Of the $160 \mathrm{gEs}$, AGN activity was suspected in 21 objects (see Section 3.1 for details). Most of the gEs studied here have X-ray halos and radio sources with luminosities lower than are typically found for cDs and BCGs. The B08 sample is taken from Rafferty et al. (2006, hereafter R06).

\section{OBSERVATIONS AND DATA ANALYSIS}

$$
\text { 3.1. X-ray }
$$


Jet powers were determined in the usual manner from the X-ray data (see B04 and R06). Gas properties used here are taken from the analysis by Jones et al. (in preparation). Cavity locations and sizes are from Nulsen et al. (in preparation), with cavity volumes and their errors calculated by the method of B04. Cavities were identified based primarily on the presence of surface brightness depressions in the X-ray emitting gas and their association with radio emission, though the latter is not requisite. To reflect this fact, each cavity system is given a figure of merit (see Section 4.1). The energy of each cavity was estimated as $4 p V$, the enthalpy of a cavity filled with relativistic gas. To estimate average cavity power, $P_{\text {cav }}$, the energy of each cavity was divided by an estimate of its age. For compatibility with B08, we give results derived assuming the age of the cavities is approximated by the buoyant rise time, $t_{\text {buoy }}$ (Churazov et al. 2001; Brüggen 2003). Because the mechanical power estimated from the cavities greatly exceeds the synchrotron power of the radio source, we assume that $P_{\text {cav }}=P_{\text {jet }}$. Further, $P_{\text {cav }}$ does not include energy channeled into shocks (e.g. Forman et al. 2007; Baldi et al. 2009a), which can exceed the energy in cavities (e.g. Nulsen et al. 2005). The cavity power estimates were summed for all the cavities in each system to obtain an estimate of the average AGN outburst power. Because the uncertainties are large (chiefly due to the uncertain volume estimates), errors are propagated in log space, assuming that the uncertainties in ambient pressure, cavity volume, and cavity age are independent of one another.

\subsection{Radio}

Radio powers, $P_{\nu_{0}}$, were estimated using the relation $P_{\nu_{0}}=$ $4 \pi D_{L}^{2}(1+z)^{\alpha-1} S_{\nu_{0}} \nu_{0}$, where $S_{\nu_{0}}$ is the flux density at the observed frequency, $\nu_{0}$, over the integrated area of the source, $z$ is redshift, $D_{L}$ is luminosity distance, and $\alpha$ is radio spectral index. The redshift correction to alpha is small for our sample of nearby objects and has been ignored. When no spectral index was available, we assumed the spectra behave as $S_{\nu} \propto \nu^{-\alpha}$ with a spectral index of $\alpha=0.8$, which is typical for extragalactic radio galaxies (Condon 1992). The $1.4 \mathrm{GHz}$ radio flux for each source was taken from the NRAO VLA Sky Survey (NVSS, Condon et al. 1998). NGC 1553 lies outside the NVSS survey area, so the $1.4 \mathrm{GHz}$ flux was estimated using the $843 \mathrm{MHz}$ Sydney University Molonglo Sky Survey (SUMSS, Bock et al. 1999) flux and $\alpha=0.89 ; \alpha$ was derived using the SUMSS and $5 \mathrm{GHz}$ Parkes (Whiteoak 1970) fluxes.

The radio morphologies for our sample are heterogeneous: some are large and extended, while others are compact. As a result, most compact sources have a single catalog entry, while large sources are divided among multiple entries. To ensure the entire radio source was measured, a fixed physical aperture of $1 \mathrm{Mpc}$ was searched around the X-ray centroid of each $\mathrm{gE}$. For each target field, all detected radio sources were overlaid on a composite image of X-ray, optical (DSS I/II), and infrared emission (2MASS). When available, the deeper and higher resolution radio data from VLA FIRST was included. A visual inspection was performed to establish which radio sources were associated with the target gE. After confirming which catalog sources are associated with the target $\mathrm{gE}$, the fluxes of the individual catalog sources were added and the associated uncertainties summed in quadrature.

Archival VLA data for each source was also reduced and analyzed. The continuum VLA data were reduced using a customized version of the NRAO VLA Archive Survey reduction pipeline. In the cases where high-resolution VLA archival data is available, multifrequency images were used to confirm the connection between NVSS detections and the host gE. Images at $1.4 \mathrm{GHz}$ were further used to check NVSS fluxes. We found flux agreement for most sources, the exceptions being IC 4296 and NGC 4782, where the NVSS flux is approximately a factor of 2 lower. The radio lobes for IC 4296 and NGC 4782 contain significant power in diffuse, extended emission which is not detected in NVSS because the NVSS flux limit is higher than the archival observations used. For these sources, the fluxes measured from the archival VLA data are used in our analysis. For the systems where nuclear radio emission was resolved from lobe emission, we found $S_{\nu_{0} \text {,nucleus }} / S_{\nu_{0} \text {, lobe }}<0.2$, suggesting the nuclear contribution to the low-resolution NVSS measurements has a small impact on our results. NGC 6269 appeared to be the exception with $S_{\nu_{0} \text {,nucleus }} / S_{\nu_{0}, \text { lobe }} \approx 1$, however, this is based upon the NVSS data only.

B08 found that using lower frequency radio data, i.e. 327 $\mathrm{MHz}$ versus $1400 \mathrm{MHz}$, resulted in a lower scatter $P_{\text {jet }}-P_{\text {radio }}$ relation. We therefore decided to test this using our sample of gEs. Unfortunately, the quality and availability of $327 \mathrm{MHz}$ data for our gE sample were not ideal, thus we gathered low-frequency radio fluxes from the CATS Database (Verkhodanov et al. 1997). The CATS Database is a compilation of more than 350 radio catalogs (e.g. WENSS, WISH, TXS, B3). For each gE, the CATS database was searched in the frequency range $200-400 \mathrm{MHz}$ for a counterpart to the NVSS and SUMSS sources. Of the $21 \mathrm{gEs}$ in our sample, 17 of them were found to have a radio source in the CATS database. CATS does not provide images for visual inspection and is composed of catalogs having a variety of spatial resolutions and flux limits. Thus, the 200-400 MHz radio powers shown in Figure 1 may include some contribution from background sources.

\section{RESULTS AND DISCUSSION \\ 4.1. $P_{\text {jet }}-P_{\text {radio }}$ Scaling Relations}

The results from the $\mathrm{X}$-ray and radio data analysis are shown in the plots of $P_{\mathrm{cav}}-P_{1.4}$ and $P_{\mathrm{cav}}-P_{200-400}$ presented in Figure 1. A figure of merit (FM) was assigned to each set of cavities through visual inspection, shown with color coding in Figure 1. To give context to each FM, we supply a wellknown cluster system as an example. FM-A cavities have well-defined boundaries and are coincident with radio emission which can be traced back to an AGN (e.g. Perseus); FM$\mathrm{B}$ cavities are coincident with radio emission from an AGN but lack well-defined boundaries (e.g. A2597); FM-C cavities have poorly-defined boundaries and their connection to AGN radio activity is unclear (e.g. A1795). FM-C cavities are excluded from all fitting, as are a subset of objects we have defined as being poorly confined (discussed in Section 4.3 and excluded from Figure 1).

Figure 1 shows a continuous, power-law relationship between cavity power and radio power spanning 8 orders of magnitude in radio power and 6 orders of magnitude in cavity power. To determine the form of the power-law relation, we performed linear fits in log-space for each frequency regime using the bivariate correlated error and intrinsic scatter (BCES) algorithm (Akritas \& Bershady 1996). The orthogonal BCES algorithm takes in asymmetric uncertainties for both variables, assumes the presence of intrinsic scatter, and performs a linear least-squares regression which minimizes the squared orthogonal distance to the best-fit relation. Param- 
eter uncertainties were calculated using 10,000 Monte Carlo bootstrap resampling trials. Our fits differ from the method used in B08 which minimized the distance in the $P_{\text {cav }}$ coordinate.

The best-fit log-space orthogonal BCES relations are:

$$
\begin{aligned}
& \log P_{\text {cav }}=0.75( \pm 0.14) \log P_{1.4}+1.91( \pm 0.18) \\
& \log P_{\text {cav }}=0.64( \pm 0.09) \log P_{200-400}+1.54( \pm 0.12)
\end{aligned}
$$

where $P_{\text {cav }}$ is in units $10^{42} \mathrm{erg} \mathrm{s}^{-1}$, and $P_{1.4}$ and $P_{200-400}$ are in units $10^{40} \mathrm{erg} \mathrm{s}^{-1}$. The scatter for each relation is $\sigma_{1.4}=0.78$ dex and $\sigma_{200-400}=0.61 \mathrm{dex}$, and the respective correlation coefficients are $r_{1.4}=0.72$ and $r_{200-400}=0.81$. We have quantified the total scatter about the best-fit relation using a weighted estimate of the orthogonal distances to the best-fit line (see Pratt et al. 2009). For comparison, the B08 scaling relations are

$$
\begin{aligned}
& \log P_{\text {cav }}=0.35( \pm 0.07) \log P_{1.4}+1.85( \pm 0.10) \\
& \log P_{\text {cav }}=0.51( \pm 0.07) \log P_{327}+1.51( \pm 0.12)
\end{aligned}
$$

where $P_{\text {cav }}$ is in units $10^{42} \mathrm{erg} \mathrm{s}^{-1}$, and $P_{1.4}$ and $P_{327}$ are in units $10^{24} \mathrm{~W} \mathrm{~Hz}^{-1}$ ( or $\approx 10^{40} \mathrm{erg} \mathrm{s}^{-1}$ ). The $\mathrm{B} 08$ relations have scatters of $\sigma_{1.4}=0.85$ dex and $\sigma_{327}=0.81$ dex. Equation 15 of B08 contains an error (Bîrzan et al. 2010), and the correct version is given in Equation 4 above.

In contrast to B08, the slopes of the relations in this work now agree to within their uncertainties. Note that we find a steeper relationship at $1.4 \mathrm{GHz}$ than B08. The difference in slope at $1.4 \mathrm{GHz}$ between our work and B08 is due to the additional data points at lower $P_{\text {jet }}$ and the different fitting method. The B08 points tend to be clumped in a fairly narrow power range, which gave the few points at the upper and lower power extremes excessive leverage over the slope. The new data extends to lower jet powers, giving a more uniform sampling and improved measurements of the slope and zero point.

The scatter in these relations is large, and B08 showed that correcting for the effect of radio aging by including a scaling with break frequency (i.e. source age) reduces the scatter by $\approx 50 \%$. The substantial scatter in the $P_{\text {jet }}-P_{\text {radio }}$ relations suggest radio lobe properties which affect synchrotron emission, e.g. age, composition, or magnetic field configuration, make synchrotron emission a poor surrogate for total jet power. Though we measure similar scatters and slopes for the gEs as the clusters, the scatter in $P_{\text {jet }}-P_{\text {radio }}$ may be particularly important for gEs, which have steeper pressure profiles and are more susceptible to disruption by AGN outbursts (Weinmann et al. 2006; Puchwein et al. 2008). We discuss this issue further in Section 4.3.

\subsection{Comparison with Models and Observational Studies}

Relations presented in Willott et al. (1999, hereafter W99) are commonly used to estimate total AGN kinetic power from observed radio power. It is therefore useful to compare our results with those of W99. For simplicity, in this section we use the parameterization $P_{\text {jet }}=\eta P_{\text {radio }}^{\Gamma}$, where $P_{\text {jet }}$ is total kinetic jet power, $\eta$ is some normalization, $\Gamma$ is a scaling index, and $P_{\text {radio }}$ is emergent synchrotron power.

W99 derive $\Gamma$ and $\eta$ using the jet model of Falle (1991) and assuming the radio lobes are at minimum energy density (see Miley 1980, for details). W99 derived $\Gamma=6 / 7(\approx 0.86)$ with $\eta \approx f^{3 / 2} 4.61 \times 10^{41} \mathrm{erg} \mathrm{s}^{-1}$ when $P_{\text {radio }}$ is in units of $10^{40} \mathrm{erg} \mathrm{s}^{-1}$. We have adjusted the fiducial W99 normalization from $151 \mathrm{MHz}$ to $1.4 \mathrm{GHz}$ assuming $S_{\nu} \propto \nu^{-0.8}$. The factor $f$ consolidates a variety of unknowns (see W99 for details). The fiducial W99 model $(f=1)$ yields $\eta$ two orders of magnitude below our normalizations, but the slopes formally agree (see Figure 2).

The W99 normalization has a weak dependence on ambient gas density. Using shallower and lower density gas profiles that are better matched to X-ray observations than the profiles adopted by W99, faster jet outflow velocities are found. Similarly, the fractional deviation from the minimum-energy condition is assumed to be small. More importantly, the W99 model depends strongly on $k$, which is the ratio of energy in non-radiating particles to relativistic electrons. We find that for $k$ lying in the range of tens to thousands, values consistent with observational findings (Dunn et al. 2005, 2006; De Young 2006; Bîrzan et al. 2008), the W99 normalization is brought into agreement with our work. The scatter in our relations may arise from intrinsic differences in radio sources (light and heavy jets), or because confined jets are born light and become heavy on large scales due to entrainment. These results suggest that typical lobes created by AGN jets contain a small fraction of their energy in relativistic electrons. Many systems showing X-ray cavities have relatively low radio powers. Systems in which a greater proportion of the energy is contained in relativistic electrons are likely to be more radio luminous and so may tend to be overrepresented in radio surveys.

For flat-spectrum, compact radio cores (i.e. small scale jets and not radio lobes), several jet models predict $\Gamma=12 / 17 \quad(\approx 0.71) \quad$ (Blandford \& Konigl 1979; Falcke \& Biermann 1995; Heinz \& Sunyaev 2003). Observational studies by Heinz \& Grimm (2005) and Merloni \& Heinz (2007), which used nuclear radio powers to estimate $P_{\text {jet }}$, found $\Gamma$ 's and $\eta$ 's consistent with our relations. The similarity of our $P_{\text {jet }}-P_{\text {radio }}$ relations with these studies may be coincidental given that our measurements are for integrated radio emission and not just nuclear radio emission. They agree, perhaps, because nuclear flux and total radio luminosity are correlated in these systems.

\subsection{Poorly Confined Sources}

The X-ray data are too shallow to image the extent of the cavities for IC 4296, NGC 315, NGC 4261, NGC 4782, and NGC 7626. Their radio lobes extend beyond the observed $\mathrm{X}$-ray halo and the radio morphologies are distinctly different from the rest of the $\mathrm{gE}$ and B08 objects. X-ray cavities typically enclose the radio emission, as is seen, for example, in M84 (Finoguenov \& Jones 2001; Finoguenov et al. 2008) where the interaction takes on some complexity (see Figure 3 ). However, the radio sources appear to be breaking through the X-ray halos of the objects listed above (see Figure 3 for an example in NGC 4261), suggesting that they may be poorly confined (PC) by their X-ray halos. While the lobes do not appear to be confined by the X-ray emitting gas, the following analysis assumes that they do remain confined by lower pressure gas. Note that the PC systems have been excluded from analysis of the $P_{\text {jet }}-P_{\text {radio }}$ relations.

To compare the properties of PC sources with the rest of our sample, we calculated $P_{\text {cav }}$ values for these systems assuming the volume of a cavity equals the volume of the corresponding radio lobe. Pressure profiles were extrapolated to large radii using $\beta$-models (Cavaliere $\&$ Fusco-Femiano 1978) fitted to the surface brightness profiles. We assumed isothermal atmospheres and a background gas pressure of $10^{-13} \mathrm{erg} \mathrm{cm}^{-3}$. The assumed background pressure is based on the mean value ob- 
served in the outskirts of clusters and groups (Cavagnolo et al. 2009). Cavity buoyancy ages cannot be directly calculated from the data. To maintain consistency with the rest of the study, $t_{\text {buoy }}$ was estimated by scaling the gas sound speed by 0.65 which is the mean value of the ratio $t_{\mathrm{cs}} / t_{\text {buoy }}$ for the B04 sample. As a result of the radio lobes extending into regions where the pressure profiles are steep and approaching the background pressure, the large lobe volumes are offset by low pressures and long ages, resulting in modest values of $P_{\text {cav }}$ relative to their radio luminosities.

Two of the PC sources, NGC 315 and NGC 4261, are in a sample of nine FR-I objects analyzed by Croston et al. (2008, hereafter C08) using XMM-Newton X-ray observations. C08 provides the $4 p V$ cavity energy and the mean temperature of the lobe environments for each FR-I source. As with the PC sources, we calculated $P_{\text {cav }}$ for each of the C08 FR-I sources using scaled sound speed. For N315 and N4261 we find no significant difference between $P_{\text {cav }}$ calculated using the Chandra data and XMM-Newton data. $P_{1.4}$ was calculated for each C08 source using the method outlined in Section 3.2. The PC and $\mathrm{C} 08$ objects are plotted in Figure 2.

In Figure 2 we highlight the location of the PC sources relative to our best-fit, $1.4 \mathrm{GHz}$ relation and the fiducial W99 relation. Figure 2 shows that the PC and C08 FR-I sources reside well below our best-fit relation. This discrepancy implies that these sources have the lowest jet power per unit radio power of all objects in the sample. One possible explanation is that these sources have lower $k$ values than the rest of the sample. This may arise naturally from matter entrainment along the jets, resulting in higher $k$ values only when a more extended, dense halo is present as is found in clusters. The radio sources in PC systems extend beyond their bright halos, and thus might not entrain significant quantities of matter. Were these sources located in denser cluster environments, they would presumably have lower radio power because a larger fraction of their energy flux would be carried in non-radiating particles. Another explanation is that PC sources are systematically more powerful than our measurements indicate due to energy being imparted to shocks. On average, shock energy is a modest correction to $P_{\text {cav }}$, factor of a few in clusters, but the incidence and variety of AGN driven shocks is broad (e.g. Kraft et al. 2003; Wise et al. 2007). However, a shock explanation would require that the fraction of jet power going into shocks is preferentially higher for systems with relatively high implied radiative efficiencies.

\section{SUMMARY AND CONCLUSIONS}

We have presented analysis of the jet power versus radio power scaling relation for the B08 sample and a sample of 21 giant elliptical galaxies observed with the $C X O$. Cavity powers were calculated for each set of cavities using similar methods to those outlined in R06. Radio powers for our sample were estimated using $1.4 \mathrm{GHz}$ and 200-400 $\mathrm{MHz}$ fluxes taken from the NVSS/SUMSS surveys and the
CATS database, respectively. We find a continuous powerlaw relation between $P_{\text {jet }}$ and $P_{\text {radio }}$ covering 6 decades in $P_{\text {radio }}$ and 8 decades in $P_{\text {jet }}$ (Figure 1). We find the power laws describing the $P_{\text {jet }}-P_{\text {radio }}$ trend have the mean form $P_{\text {jet }} \approx$ $5.8 \times 10^{43}\left(P_{\text {radio }} / 10^{40}\right)^{0.70} \mathrm{erg} \mathrm{s}^{-1}$, and a scatter about the fit of $\approx 0.7$ dex. Our relations agree reasonably well with previous observational studies and they are consistent with theoretical expectations if the fraction of the energy in relativistic electrons is small in a typical radio lobe.

Several groups have applied the Bîrzan scaling relations to study the effects of AGN feedback on structure formation, e.g. Best et al. (2007) and Magliocchetti \& Brüggen (2007), with some groups now suggesting that distributed low-power radio galaxies may dominate heating of the intracluster medium, e.g. Hart et al. (2009). Up to now, the available observational results, which were primarily calibrated to high-power radio sources, did not clearly indicate if a $P_{\text {jet }}-P_{\text {radio }}$ relation would be continuous, or of comparable scatter, for lower power radio sources. Assuming there is no redshift evolution of $P_{\text {jet }}{ }^{-}$ $P_{\text {radio, }}$ our relations suggest higher mass galaxies dominate over lower mass galaxies in the process of mechanical heating within clusters and groups.

The subset of objects with radio sources that are poorly confined by their hot halo have $P_{\text {radio }} / P_{\text {jet }}$ ratios which are large relative to the rest of our sample (see Figure 2). In addition, PC sources reside in the same region of the $P_{\text {jet }}-P_{\text {radio }}$ plane as the FR-I sources taken from C08. Radio emission from lobes depends on their composition, so that the large scatter in the $P_{\text {jet }}-P_{\text {radio }}$ relationship may result from processes such as gas entrainment and shocks. It seems likely that some of the scatter arises as radio sources age, but it remains unclear what other factors are important. With better constraints on the relationships between jet power and radio synchrotron power, our understanding of the composition and mechanical power of radio sources has improved considerably. Models and simulations of AGN feedback that successfully reproduce these results will yield new insight into jet formation mechanisms and the structure of the central engine.

KWC and BRM acknowledge generous support from the Natural Sciences and Engineering Research Council of Canada and grants from the $C X O$. CJ thanks the Smithsonian Institution for generous support. $\mathrm{PN}$ thanks the $C X O$ Center for supporting this work. KWC thanks Judith Croston, David Rafferty, Lorant Sjouwerman, and Chris Willott for helpful discussions. We also thank the anonymous referee for a prompt and helpful review. The $C X O$ Center is operated by the Smithsonian Astrophysical Observatory for and on behalf of NASA under contract NAS8-03060. The National Radio Astronomy Observatory is a facility of the National Science Foundation operated under cooperative agreement by Associated Universities, Inc.

Facilities: CXO (ACIS) VLA

\section{REFERENCES}

Akritas, M. G., \& Bershady, M. A. 1996, ApJ, 470, 7062

Allen, S. W., Dunn, R. J. H., Fabian, A. C., Taylor, G. B., \& Reynolds, C. S. 2006, MNRAS, 372, 216

Baldi, A., Forman, W., Jones, C., Kraft, R., Nulsen, P., Churazov, E., David, L., \& Giacintucci, S. 2009a, ApJ, 707, 1034 2, 6

Baldi, A., Forman, W., Jones, C., Nulsen, P., David, L., Kraft, R., \& Simionescu, A. 2009b, ApJ, 694, 4796

Best, P. N., von der Linden, A., Kauffmann, G., Heckman, T. M., \& Kaiser, C. R. 2007, MNRAS, 379, 8941,4
Beuing, J., Dobereiner, S., Böhringer, H., \& Bender, R. 1999, MNRAS, 302, 2091

Biller, B. A., Jones, C., Forman, W. R., Kraft, R., \& Ensslin, T. 2004, ApJ, 613,2386

Bîrzan, L., McNamara, B. R., Nulsen, P. E. J., Carilli, C. L., , \& Wise, M. W. 2010, The Astrophysical Journal, 709, 5463

Bîrzan, L., McNamara, B. R., Nulsen, P. E. J., Carilli, C. L., \& Wise, M. W. 2008, ApJ, 686, 859 1, 3 
Bîrzan, L., Rafferty, D. A., McNamara, B. R., Wise, M. W., \& Nulsen, P. E. J. 2004, ApJ, 607, 8001

Blandford, R. D., \& Konigl, A. 1979, ApJ, 232, 343

Blanton, E. L., Sarazin, C. L., \& Irwin, J. A. 2001, ApJ, 552, 1066

Bock, D. C.-J., Large, M. I., \& Sadler, E. M. 1999, AJ, 117, 15782

Bower, R. G., Benson, A. J., Malbon, R., Helly, J. C., Frenk, C. S., Baugh, C. M., Cole, S., \& Lacey, C. G. 2006, MNRAS, 370, 6451

Brüggen, M. 2003, ApJ, 592, 8392

Cavagnolo, K. W., Donahue, M., Voit, G. M., \& Sun, M. 2009, ApJS, 182, 124

Cavaliere, A., \& Fusco-Femiano, R. 1978, A\&A, 70, 6773

Churazov, E., Brüggen, M., Kaiser, C. R., Böhringer, H., \& Forman, W. 2001, ApJ, 554, 261 1, 2

Condon, J. J. 1992, ARA\&A, 30, 5752

Condon, J. J., Cotton, W. D., Greisen, E. W., Yin, Q. F., Perley, R. A., Taylor, G. B., \& Broderick, J. J. 1998, AJ, 115, 16932

Croston, J. H., Hardcastle, M. J., Birkinshaw, M., Worrall, D. M., \& Laing, R. A. 2008, MNRAS, 386, 1709 4, 7

Croton, D. J., Springel, V., White, S. D. M., De Lucia, G., Frenk, C. S., Gao, L., Jenkins, A., Kauffmann, G., Navarro, J. F., \& Yoshida, N. 2006, MNRAS, 365, 111

David, L. P., Jones, C., Forman, W., Nulsen, P., Vrtilek, J., O’Sullivan, E., Giacintucci, S., \& Raychaudhury, S. 2009, ApJ, 705, 6246

David, L. P., Jones, C., Forman, W., Vargas, I. M., \& Nulsen, P. 2006, ApJ, 653, 2076

De Young, D. S. 2006, ApJ, 648, 2003

De Young, D. S., O’Neill, S. M., \& Jones, T. W. 2008, in Astronomical Society of the Pacific Conference Series, Vol. 386, Extragalactic Jets: Theory and Observation from Radio to Gamma Ray, ed. T. A. Rector \& D. S. De Young, 343-+ 1

Diehl, S., \& Statler, T. S. 2008, ApJ, 687, 9866

Dunn, R. J. H., Fabian, A. C., \& Celotti, A. 2006, MNRAS, 372, 17413

Dunn, R. J. H., Fabian, A. C., \& Taylor, G. B. 2005, MNRAS, 364, 1343 1, 3

Fabian, A. C., Sanders, J. S., Ettori, S., Taylor, G. B., Allen, S. W., Crawford, C. S., Iwasawa, K., Johnstone, R. M., \& Ogle, P. M. 2000, MNRAS, 318, L65 1

Falcke, H., \& Biermann, P. L. 1995, A\&A, 293, 6653

Falle, S. A. E. G. 1991, MNRAS, 250, 5813

Finoguenov, A., \& Jones, C. 2001, ApJ, 547, L107 3

Finoguenov, A., Ruszkowski, M., Jones, C., Brüggen, M., Vikhlinin, A., \& Mandel, E. 2008, ApJ, 686, 9113

Forman, W., Jones, C., Churazov, E., Markevitch, M., Nulsen, P., Vikhlinin, A., Begelman, M., Böhringer, H., Eilek, J., Heinz, S., Kraft, R., Owen, F., \& Pahre, M. 2007, ApJ, 665, 10572

Hart, Q. N., Stocke, J. T., \& Hallman, E. J. 2009, ApJ, 705, 8544

Heinz, S., \& Grimm, H. J. 2005, ApJ, 633, 3843

Heinz, S., \& Sunyaev, R. A. 2003, MNRAS, 343, L59 3

Jetha, N. N., Ponman, T. J., Hardcastle, M. J., \& Croston, J. H. 2007, MNRAS, 376, 1936

Kauffmann, G., \& Haehnelt, M. 2000, MNRAS, 311, 5761

Kormendy, J., \& Richstone, D. 1995, ARA\&A, 33, 5811

Kraft, R. P., Forman, W. R., Churazov, E., Laslo, N., Jones, C., Markevitch, M., Murray, S. S., \& Vikhlinin, A. 2004, ApJ, 601, 2216
Kraft, R. P., Vázquez, S. E., Forman, W. R., Jones, C., Murray, S. S., Hardcastle, M. J., Worrall, D. M., \& Churazov, E. 2003, ApJ, 592, 1294

Lanz, L., Jones, C., Forman, W. R., Ashby, M. L. N., Kraft, R., \& Hickox, R. C. 2010, in Bulletin of the American Astronomical Society, Vol. 41, Bulletin of the American Astronomical Society, 483-+ 6

Machacek, M., Nulsen, P. E. J., Jones, C., \& Forman, W. R. 2006, ApJ, 648, 9476

Machacek, M. E., Kraft, R. P., Jones, C., Forman, W. R., \& Hardcastle, M. J. 2007, ApJ, 664, 8046

Magliocchetti, M., \& Brüggen, M. 2007, MNRAS, 379, 2604

Magorrian, J., Tremaine, S., Richstone, D., Bender, R., Bower, G., Dressler, A., Faber, S. M., Gebhardt, K., Green, R., Grillmair, C., Kormendy, J., \& Lauer, T. 1998, AJ, 115, 22851

Mathur, S., Stoll, R., Krongold, Y., Nicastro, F., Brickhouse, N., \& Elvis, M. 2009, ArXiv e-prints: 0910.36911

McNamara, B. R., Wise, M., Nulsen, P. E. J., David, L. P., Sarazin, C. L., Bautz, M., Markevitch, M., Vikhlinin, A., Forman, W. R., Jones, C., \& Harris, D. E. 2000, ApJ, 534, L135 1

Merloni, A., \& Heinz, S. 2007, MNRAS, 381, 5893

Miley, G. 1980, ARA\&A, 18, 1653

Nulsen, P. E. J., Hambrick, D. C., McNamara, B. R., Rafferty, D., Bîrzan, L., Wise, M. W., \& David, L. P. 2005, ApJ, 625, L9 2

O’Sullivan, E., Ponman, T. J., \& Collins, R. S. 2003, MNRAS, 340, 13751

Pellegrini, S., Venturi, T., Comastri, A., Fabbiano, G., Fiore, F., Vignali, C., Morganti, R., \& Trinchieri, G. 2003, ApJ, 585, 6776

Pratt, G. W., Croston, J. H., Arnaud, M., \& Böhringer, H. 2009, A\&A, 498, 3613

Puchwein, E., Sijacki, D., \& Springel, V. 2008, ApJ, 687, L53 3

Rafferty, D. A., McNamara, B. R., Nulsen, P. E. J., \& Wise, M. W. 2006 ApJ, 652, 2161

Randall, S. W., Forman, W. R., Giacintucci, S., Nulsen, P. E. J., Sun, M., Jones, C., Churazov, E., David, L. P., Kraft, R., Donahue, M., Blanton, E. L., Simionescu, A., \& Werner, N. 2010, ArXiv e-prints 6

Randall, S. W., Jones, C., Kraft, R., Forman, W. R., \& O’Sullivan, E. 2009, ApJ, 696, 14316

Silk, J., \& Rees, M. J. 1998, A\&A, 331, L1 1

Sivakoff, G. R., Sarazin, C. L., \& Carlin, J. L. 2004, ApJ, 617, 2626

Sun, M. 2009, ApJ, 704, 15866

Verkhodanov, O. V., Trushkin, S. A., Andernach, H., \& Chernenkov, V. N. 1997, in Astronomical Society of the Pacific Conference Series, Vol. 125, Astronomical Data Analysis Software and Systems VI, ed. G. Hunt \& H. Payne, 322-+ 2

Weinmann, S. M., van den Bosch, F. C., Yang, X., Mo, H. J., Croton, D. J., \& Moore, B. 2006, MNRAS, 372, 11613

Whiteoak, J. B. 1970, Astrophys. Lett., 5, 292

Willott, C. J., Rawlings, S., Blundell, K. M., \& Lacy, M. 1999, MNRAS, $309,10171,3,7$

Wise, M. W., McNamara, B. R., Nulsen, P. E. J., Houck, J. C., \& David, L. P. 2007, ApJ, 659, 11534

Worrall, D. M., Birkinshaw, M., \& Hardcastle, M. J. 2003, MNRAS, 343, L73 6

Xu, H., Gu, J., Gu, L., Zhang, Z., Wang, Y., \& An, T. 2010, Research in Astronomy and Astrophysics, 10, 2206

Zezas, A., Birkinshaw, M., Worrall, D. M., Peters, A., \& Fabbiano, G. 2005, ApJ, 627, 7116 
TABLE 1

SUMMARY OF SAMPLE

\begin{tabular}{|c|c|c|c|c|c|c|c|c|c|}
\hline $\begin{array}{c}\text { Source } \\
- \\
(1)\end{array}$ & $\begin{array}{l}\text { R.A. } \\
\text { h:m:s } \\
(2)\end{array}$ & $\begin{array}{l}\text { Dec. } \\
\circ:{ }^{\circ}:{ }^{\prime \prime} \\
(3)\end{array}$ & $\begin{array}{c}D_{L} \\
\mathrm{Mpc} \\
(4)\end{array}$ & $\begin{array}{c}P_{1.4} \\
10^{39} \mathrm{erg} \mathrm{s}^{-1} \\
(5)\end{array}$ & $\begin{array}{c}P_{200-400} \\
10^{39} \mathrm{erg} \mathrm{s}^{-1} \\
(6)\end{array}$ & $\begin{array}{c}P_{\text {cav }}^{\text {buoy }} \\
10^{42} \mathrm{erg} \mathrm{s}^{-1} \\
(7)\end{array}$ & $\begin{array}{c}L_{X}\left(<r_{\text {cool }}\right) \\
10^{33} \mathrm{erg} \mathrm{s}^{-1} \\
\quad(8)\end{array}$ & $\begin{array}{l}\text { FM } \\
- \\
(9)\end{array}$ & $\begin{array}{c}\text { Ref. } \\
- \\
(10)\end{array}$ \\
\hline IC $4296^{\dagger}$ & $13: 36: 39$ & $-33: 57: 57$ & 54.3 & $38.9 \pm 3.5$ & $190 \pm 12$ & $3.87_{-3.03}^{+1.44}$ & 18.6 & B & [1] \\
\hline NGC 193 & $00: 39: 19$ & $+03: 19: 52$ & 63.5 & $8.60 \pm 0.27$ & $7.98 \pm 0.40$ & $9.99_{-4.54}^{+3.03}$ & 45.7 & A & [2] \\
\hline NGC $315^{\dagger}$ & $00: 57: 49$ & $+30: 21: 09$ & 73.1 & $59.2 \pm 5.4$ & $21.5 \pm 1.5$ & $6.58_{-4.96}^{+2.54}$ & 28.8 & B & [3] \\
\hline NGC 507 & $01: 23: 40$ & $+33: 15: 20$ & 83.9 & $1.16 \pm 0.05$ & $1.85 \pm 0.19$ & $19.9_{-6.4}^{+4.46}$ & 1099 & B & [4] \\
\hline NGC 777 & $02: 00: 15$ & $+31: 25: 46$ & 63.6 & $0.0473 \pm 0.0034$ & $0.0376 \pm 0.0038$ & $\begin{array}{r}-6.8 \\
4.08_{-3.03}^{+1.48}\end{array}$ & 98.5 & $\mathrm{C}$ & [5] \\
\hline NGC 1316 & $03: 22: 42$ & $-37: 12: 30$ & 21.6 & $0.198 \pm 0.008$ & $0.0476 \pm 0.0081$ & $\begin{array}{l}1.11_{-0.40}^{+0.23} \\
1.26\end{array}$ & 5.93 & B & [6] \\
\hline NGC 1553 & $04: 16: 11$ & $-55: 46: 49$ & 18.6 & $0.0039 \pm 0.0006$ & $0.202 \pm 0.067$ & $\begin{array}{l}-0.40 \\
0.98_{-0.70}^{+0.34}\end{array}$ & 172 & $\mathrm{C}$ & [7] \\
\hline NGC 1600 & 04:31:40 & $-05: 05: 10$ & 58.0 & $0.346 \pm 0.015$ & $\ldots$ & $\begin{array}{r}-0.70 \\
1.87_{-200}^{+0.82}\end{array}$ & 11.6 & B & [8] \\
\hline NGC 3608 & $11: 16: 59$ & $+18: 08: 55$ & 23.0 & $<0.0022$ & $0.0336 \pm 0.0002$ & $0.05_{-0.03}^{+0.00}$ & 0.783 & $\mathrm{C}$ & [9] \\
\hline NGC $4261^{\dagger}$ & $12: 19: 23$ & $+05: 49: 31$ & 31.8 & $17.7 \pm 0.5$ & $61.4 \pm 6.5$ & $0.91_{-0.79}^{+0.03}$ & 7.57 & B & [10] \\
\hline NGC 4374 & $12: 25: 04$ & $+12: 53: 13$ & 18.5 & $3.47 \pm 0.12$ & $2.72 \pm 0.28$ & $5.03_{-5.53}^{+2.17}$ & 7.58 & A & [11] \\
\hline NGC 4472 & $12: 29: 47$ & +08:00:02 & 16.4 & $0.115 \pm 0.005$ & $0.0914 \pm 0.0131$ & $\begin{array}{r}-5.53 \\
0.53_{-0.32}^{+0.16}\end{array}$ & 16.1 & A & [12] \\
\hline NGC 4552 & $12: 35: 40$ & $+12: 33: 23$ & 15.4 & $0.0398 \pm 0.0012$ & $\ldots$ & $\begin{array}{l}-0.32 \\
0.53_{-0.17}^{+0.12}\end{array}$ & 2.22 & A & [13] \\
\hline NGC 4636 & $12: 42: 50$ & $+02: 41: 16$ & 14.7 & $0.0281 \pm 0.0010$ & $0.0843 \pm 0.0005$ & $2.76_{-0.91}^{+0.56}$ & 37.6 & B & [14] \\
\hline NGC $4782^{\dagger}$ & $12: 54: 36$ & $-12: 34: 07$ & 66.9 & $57.6 \pm 1.8$ & $66.8 \pm 6.7$ & $2.43_{-1.43}^{+0.86}$ & 13.9 & B & [15] \\
\hline NGC 5044 & $13: 15: 24$ & $-16: 23: 08$ & 31.4 & $0.0571 \pm 0.0018$ & $0.0126 \pm 0.0013$ & $4.18_{-1.97}^{+1.18}$ & 425 & B & [16] \\
\hline NGC 5813 & $15: 01: 11$ & $+01: 42: 07$ & 32.4 & $0.0260 \pm 0.0018$ & $0.334 \pm 0.054$ & $3.97_{-2.36}^{+1.02}$ & 180 & A & [17] \\
\hline NGC 5846 & $15: 06: 29$ & $+01: 36: 20$ & 24.9 & $0.0220 \pm 0.0014$ & $0.0609 \pm 0.0001$ & $0.88_{-0.59}^{+0.30}$ & 64.4 & B & [18] \\
\hline NGC 6269 & $16: 57: 58$ & $+27: 51: 16$ & 155 & $1.99 \pm 0.08$ & $\ldots$ & $1.54_{-1.03}^{+0.39}$ & 118 & $\mathrm{C}$ & [19] \\
\hline NGC 6338 & $17: 15: 23$ & $+57: 24: 40$ & 120 & $1.37 \pm 0.04$ & $0.451 \pm 0.045$ & $11.0_{-69}^{+3.33}$ & 388 & B & [20] \\
\hline NGC $7626^{\dagger}$ & $23: 20: 43$ & $+08: 13: 01$ & 51.7 & $3.76 \pm 0.14$ & $\ldots$ & $\begin{array}{r}-6.9 \\
0.39_{-0.18}^{+0.09}\end{array}$ & 11.5 & B & [21] \\
\hline
\end{tabular}

Note. - Col. (1) Source name - those with a dagger $(\dagger)$ are poorly confined (see Section 4.3) and are not plotted in Figure 1; Col. (2) Right ascension; Col. (3) Declination; Col. (4) Luminosity distance; Col. (5) $1.4 \mathrm{GHz}$ radio power; Col. (6) 200-400 MHz radio power; Col. (7) Cavity power calculated using buoyancy age; Col. (8) $0.3-2.0 \mathrm{keV}$ X-ray luminosity within radius where $t_{\text {cool }}<7.7$ Gyrs; Col. (9) Cavity system figure of merit (see Section 4). Col. (10) References for other relevant studies: [1] Pellegrini et al. (2003), [2] Diehl \& Statler (2008), [3] Worrall et al. (2003), [4] Kraft et al. (2004), [5] Sun (2009), [6] Lanz et al. (2010), [7] Blanton et al. (2001), [8] Sivakoff et al. (2004), [9] David et al. (2006), [10] Zezas et al. (2005), [11] Xu et al. (2010), [12] Biller et al. (2004), [13] Machacek et al. (2006), [14] Baldi et al. (2009a), [15] Machacek et al. (2007), [16] David et al. (2009), [17] Randall et al. (2010), [18] Allen et al. (2006), [19] Baldi et al. (2009b), [20] Jetha et al. (2007), [21] Randall et al. (2009), 

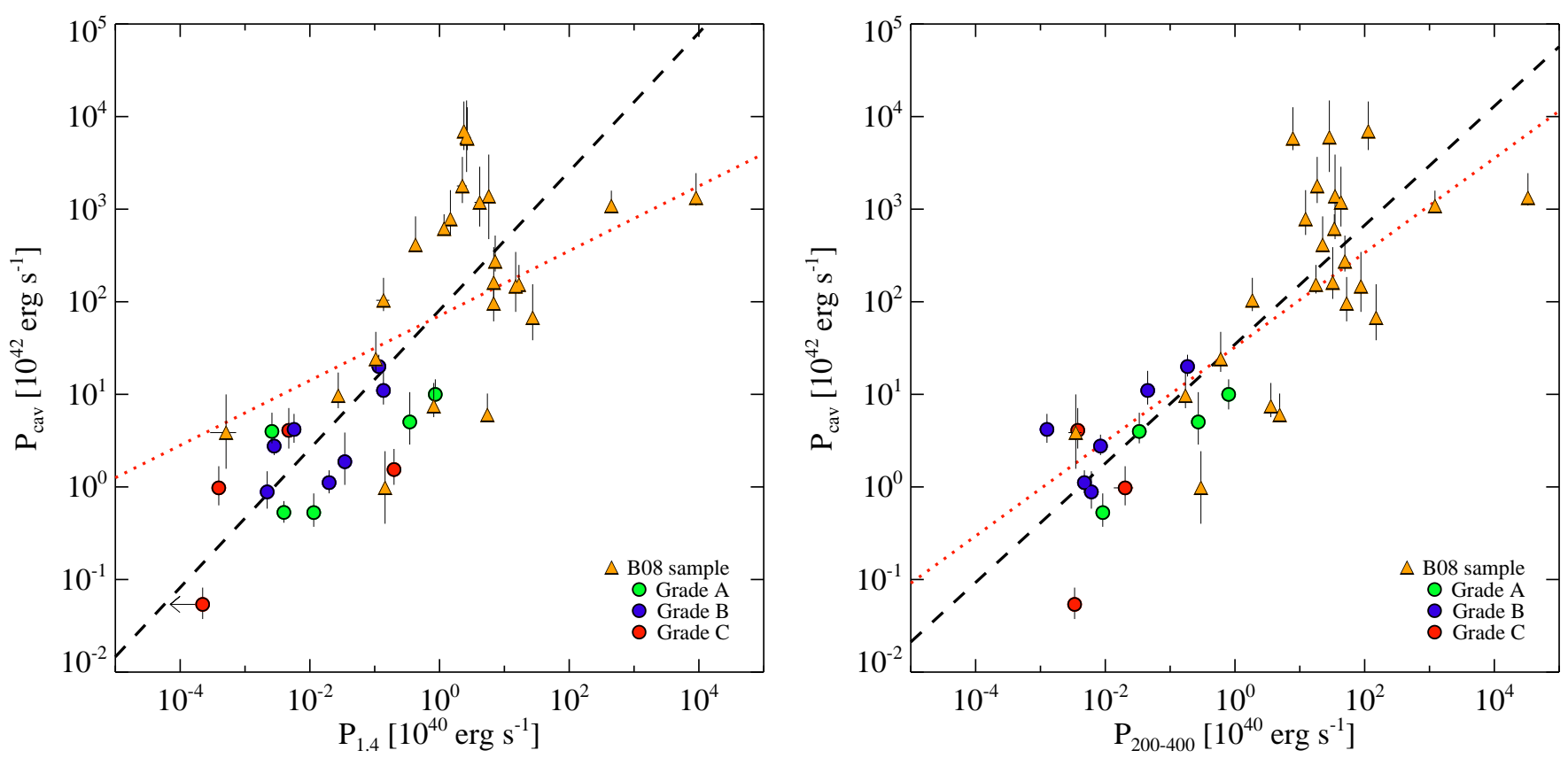

FIG. 1.- Cavity power vs. radio power. Orange triangles represent the galaxy clusters and groups sample from B08. Filled circles represent our sample of gEs with colors representing the cavity system figure of merit (see Section \$3.1): green = 'A,' blue = 'B,' and red = 'C.' The dotted red lines represent the best-fit power-law relations presented in B08 using only the orange triangles. The dashed black lines represent our BCES best-fit power-law relations. Left: Cavity power vs. $1.4 \mathrm{GHz}$ radio power. Right: Cavity power vs. 200-400 MHz radio power.

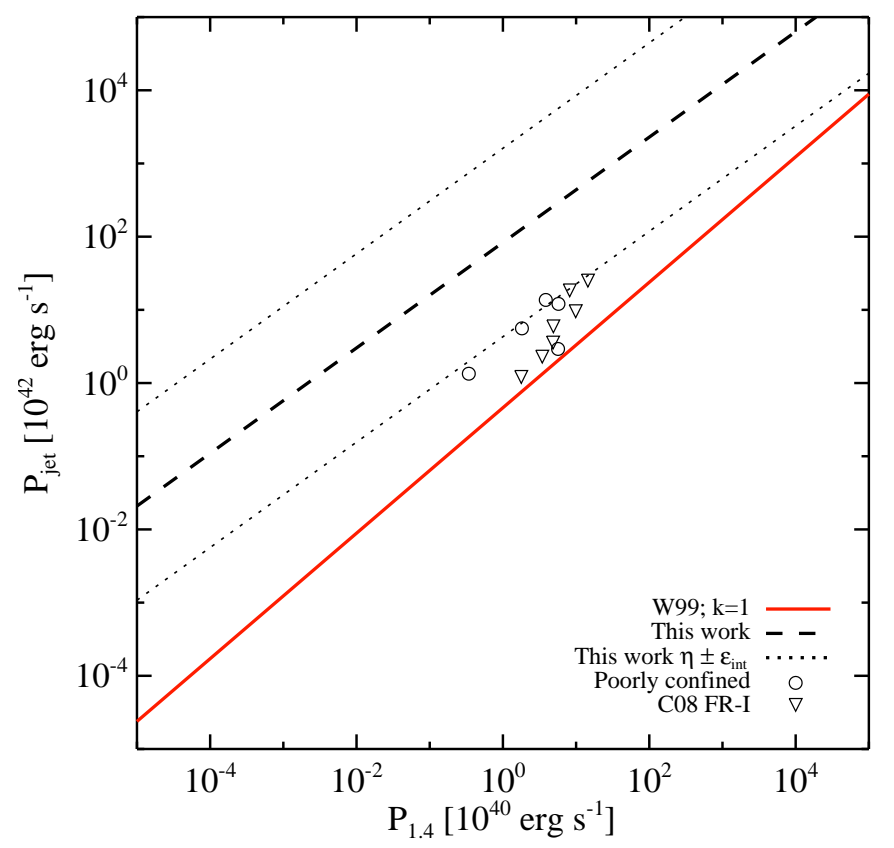

FIG. 2.- Comparison of scaling relations between jet power and radio luminosity. The solid red line represents the Willott et al. (1999, W99) model with $k=1$. The dashed black line is our best-fit $P_{\text {jet }}-P_{1.4}$ relation (Equation 1). The dotted black lines denote the upper and lower limits of our best-fit relation after including intrinsic scatter of $\epsilon_{\text {int }}=1.3$ dex. The unfilled black circles denote the poorly confined sources discussed in Section 4.3, and the downfacing black triangles are FR-I sources taken from the sample in Croston et al. (2008, C08). 
K. W. Cavagnolo et al.

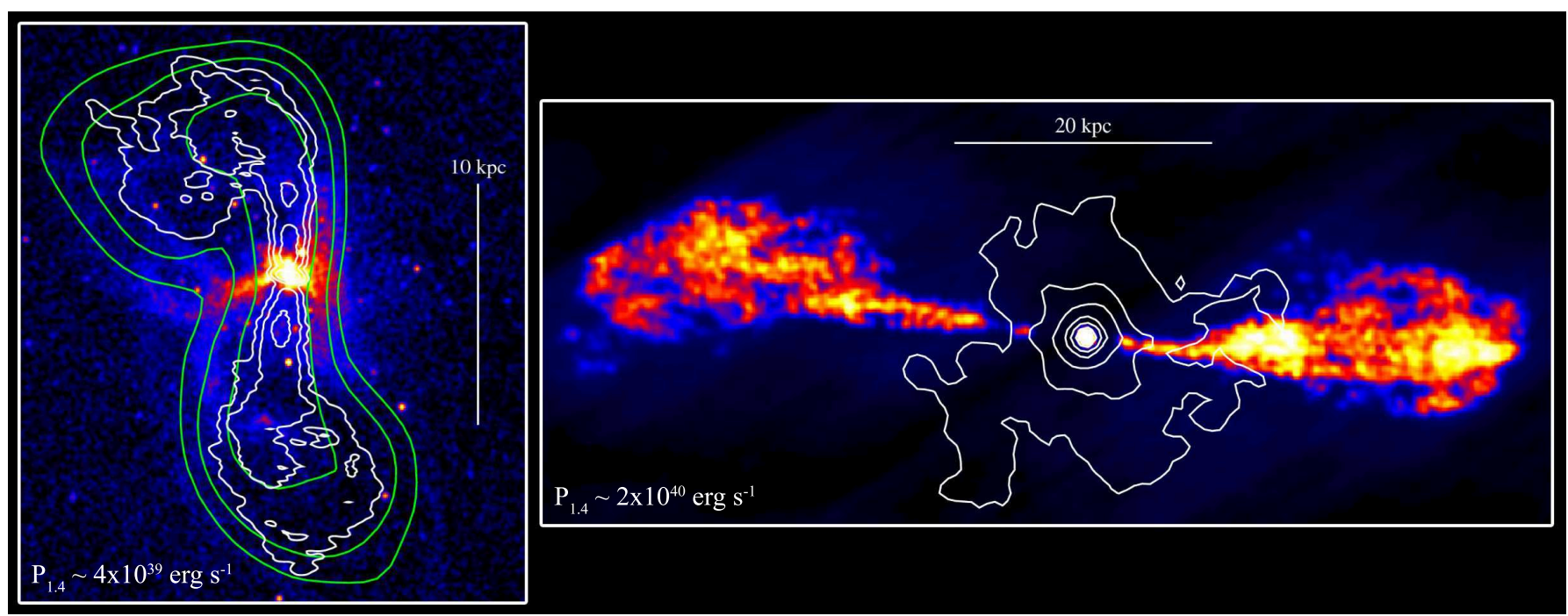

FIG. 3.-Left: Chandra X-ray image of the giant elliptical M84 (NGC 4374). Contours trace out $1.4 \mathrm{GHz}$ radio emission as observed with VLA C-configuration (green) and $\mathrm{AB}$-configuration (white) ranging from $\approx 0.5-50 \mathrm{mJy}$ in log spaced steps of $10 \mathrm{mJy}$. Note the displacement of the $\mathrm{X}$-ray gas around the bipolar AGN jet outflows. M84 examplifies the typical interaction between an AGN outflow and a hot gaseous halo. Right: VLA B-configuration 1.4 GHz radio image of the AGN jets eminating from the giant elliptical NGC 4261. White contours trace Chandra observed X-ray emission of the hot halo surrounding N4261. The contours cover the surface brightness range of $\approx 5-50 \mathrm{cts} \mathrm{arcsec}^{-2}$ in linear spaced steps of $5 \mathrm{cts} \operatorname{arcsec}^{-2}$. N4261 demonstrates the characteristic traits of what we have termed poorly confined sources: a compact X-ray halo, small centralized cavities along the jets, FR-I-like radio morphology, and pluming beyond the "edge" of the X-ray halo. 